\title{
Dietary behaviour and health in Northern Ireland: an exploration of biochemical and haematological associations
}

\author{
M E Barker, S I McClean, J J Strain, K A Thompson
}

\begin{abstract}
Study objective-The aim was to determine the relationships between dietary behaviour and biochemical and haematological measures.
\end{abstract}

Design-This was a cross sectional population study.

Setting-The study took place in the general community within Northern Ireland.

Subjects-522 randomly selected adults aged 18-64 years took part $(65 \%$ of the eligible sample).

Measurements and main results-Four dietary behaviours were identified using principal components analysis from $7 \mathrm{~d}$ weighed dietary records described in terms of mean intake of 41 food groups. Haematological and biochemical analyses were carried out on non-fasting blood samples. Social, personal, and lifestyle information was ascertained through interviewer administered questionnaires. Partial correlations controlled for age and smoking behaviour were calculated. There were significant negative associations between iron status measures and the "traditional" behaviour, while the "meat and two veg" behaviour showed positive associations. There were positive associations between the "cosmopolitan" and "convenience" behaviours and folate status in women. White cell count and platelet levels in women were negatively associated with the "traditional" and "cosmopolitan" behaviours. Total cholesterol levels showed significant negative associations with the "cosmopolitan" behaviour in women and HDL cholesterol levels were positively associated with the "cosmopolitan", "convenience" and "meat and two veg" behaviours.

Conclusions-Through a multivariate approach to dietary assessment it is possible to identify food combinations that cluster and interact to influence biochemical and haematological indices of health status.

The Northern Ireland Diet, Lifestyle and Health Study ${ }^{1}$ was primarily undertaken to obtain baseline measures of nutritional status of a random cross section of the population of Northern Ireland. The study has provided nutrient intake, haematological, and biochemical data, as well as extensive information on the demographic, lifestyle, anthropometric, and psychological characteristics of the population. ${ }^{1}$ Further analyses ${ }^{2}$ provided information on individual dietary behaviours in relation to sociocultural demographics, lifestyle habits, and anthropometric measures. The focus of the present study is to relate these individual dietary behaviours to nutritional health as defined by a range of biochemical and haematological measures. Hence, this study assesses how food combinations (generated using principal components analysis from $7 \mathrm{~d}$ weighed intake information in terms of $\mathbf{4 1}$ food groups), rather than specific foods or nutrients, affect levels of key biochemical and haematological health indices.

This approach has been used previously in the USA $^{34}$ in an analysis of dietary information from the Ten-State Survey, the National Health and Nutrition Examination Survey (NHANES 1), and the National Food Consumption Survey (NFCS). However, no comparative information is available for Northern Ireland or indeed Great Britain. The US studies ${ }^{34}$ identified seven distinct eating patterns using exploratory factor analysis and found that certain eating patterns were associated with an absence of both clinical symptoms and nutritional deficiencies as defined by biochemical indices. For instance, using NFCS data to identify dietary patterns and comparing those patterns to biochemical measures and clinical symptoms, it was shown that consumers of a pattern identified as having more dairy produce and soups and less sugary foods/beverages than other patterns, had fewer biochemical abnormalities and clinical symptoms. More recently, in the Bogalusa Heart Study of adolescent and young adults, Nicklas et $a l^{5}$ illustrated the potential of using an eating pattern model to explore the relationships between diet and cardiovascular risk factors.

The four major dietary patterns that have been identified in Northern Ireland ${ }^{2}$ were a "traditional" diet, a "cosmopolitan" diet, a "convenience" diet, and a "meat and two veg" diet. The "traditional" diet was characterised by the predominance of staple, indigenous, core foods such as bread, tea and coffee, spreading fats, cakes and desserts, potatoes, milk, preserves, eggs, bacon, and vegetables. Conversely, chips, wines, liqueurs and spirits, and rice and pasta were avoided. The "cosmopolitan" diet was a more diverse regimen; the predominant foods in this dietary behaviour were fruit, vegetables, rice and pasta, egg and cheese dishes, cream, cheese, tea and coffee, patés, breakfast cereals, fatty fish, and shellfish. Also within this regimen foods such as chips, sausages, peas and beans, savoury pies and beer, which could be considered to have either prosaic or health threatening connotations, 
were avoided. The third dietary behaviour was orientated towards a regimen of fast and convenience food such as beer, chips, sauces, soft drinks, nuts, cheese, rice and pasta, savoury pies and cooked meat dishes. The final dietary behaviour identified, the "meat and two veg" diet, was as its name suggests characterised by the predominance of meat and vegetables, while the consumption of sauces, potatoes, wines, liqueurs and spirits, white fish, fatty fish, and shellfish also comprised elements of this behaviour.

\begin{abstract}
Methods
A detailed account of the sampling methods, design of instruments for data collection and methods of data collection has been provided elsewhere. ${ }^{1}$ The study was based on a two stage sample; first of Northern Ireland households, and second of individuals from sampled households. The method of $\mathrm{Kish}^{6}$ was used to extract a sample of 797 eligible subjects aged between 16 and 64 years who reflected the sex and age distribution of the population. Of these, 616 subjects completed the full range of study measures and 592 subjects (74\% of the eligible sample) completed the weighed record of food intake.

Non-fasted venous blood samples (taken only in the evening) were obtained from 522 subjects (230 men and 292 women) aged 18 to 64 years, representing $65 \%$ of the eligible sample. Blood samples were processed immediately and the serum or plasma separated. Whole blood and serum were cooled and subsequently analysed at the Royal Victoria Hospital Laboratories, Belfast, which subscribe to the UK National external quality assessment scheme. A full haematological profile was obtained with an automated technique using a Coulter counter. Serum ferritin was measured by radioimmunoassay using antibodies raised to human spleen ferritin labelled with ${ }^{125}$ iodine reagent $^{7}$ supplied by Amersham International, Amersham, Bucks, England. Transferrin saturation was calculated by expressing serum $\mathrm{Fe}$ as a percentage of total $\mathrm{Fe}$ binding capacity (TIBC). Measurement of serum
\end{abstract}

Table I Partial correlation coefficients (controlled for age and smoking habit) between dietary behaviours and blood measurements ${ }^{\mathrm{a}}$ of iron, folate and vitamin B-12 status for men and women

\begin{tabular}{|c|c|c|c|c|}
\hline & \multicolumn{4}{|c|}{ Dietary behaviour } \\
\hline & "Traditional" & “Cosmopolitan" & "Convenience" & $\begin{array}{l}\text { "Meat and } \\
\text { two Veg" }\end{array}$ \\
\hline & \multicolumn{4}{|c|}{ Men } \\
\hline Haemoglobin & $-0 \cdot 12^{\star}$ & 0.00 & -0.02 & 0.04 \\
\hline SF & $-0.18 \dagger$ & 0.03 & 0.09 & $0.22 \dagger$ \\
\hline TS & -0.02 & 0.01 & $0 \cdot 10$ & $0 \cdot 11$ \\
\hline $\mathrm{MCHC}$ & 0.06 & -0.01 & 0.07 & -0.02 \\
\hline PCV & $-0 \cdot 10$ & -0.00 & $-0 \cdot 15^{\star}$ & -0.09 \\
\hline MCV & 0.03 & 0.09 & -0.05 & 0.04 \\
\hline Red cell folate & -0.05 & -0.08 & 0.08 & -0.09 \\
\hline Serum folate & 0.03 & -0.06 & 0.07 & 0.03 \\
\hline \multirow[t]{2}{*}{ Serum vitamin B-12 } & -0.03 & 0.05 & 0.06 & $0 \cdot 14^{\star}$ \\
\hline & \multicolumn{4}{|c|}{ Women } \\
\hline Haemoglobin & -0.06 & 0.03 & 0.07 & -0.01 \\
\hline SF & -0.05 & $-0 \cdot 10$ & 0.05 & 0.02 \\
\hline TS & $-0 \cdot 12^{\star}$ & $0 \cdot 12^{\star}$ & $0 \cdot 11^{\star}$ & 0.08 \\
\hline MCHC & -0.00 & 0.05 & 0.06 & -0.01 \\
\hline PCV & 0.07 & $-0 \cdot 11^{\star}$ & $-0 \cdot 13^{\star}$ & $0.17 \dagger$ \\
\hline $\mathrm{MCV}$ & -0.03 & -0.06 & -0.00 & 0.04 \\
\hline Red cell folate & 0.05 & $0 \cdot 11^{\star}$ & $0 \cdot 12^{\star}$ & -0.01 \\
\hline Serum folate & 0.06 & $0.18 t$ & $0.15 \dagger$ & -0.01 \\
\hline Serum vitamin B-12 & 0.05 & $0 \cdot 18 t$ & $0.18 t$ & 0.05 \\
\hline
\end{tabular}

$\mathrm{Fe}$, after reduction and formation of a blue complex with mercuric tripyridyltirazine, and TIBC by calculation from the unbound $\mathrm{Fe}$ remaining after the addition of a known amount of excess ferrous $\mathrm{Fe}$, were performed on the American monitor "parallel" system. Mean corpuscular haemoglobin concentration (MCHC) was the haemoglobin:packed cell volume ratio, expressed as a percentage. Serum and red cell folate and serum vitamin B-12 were analysed with a dual radioimmunoassay kit (using ${ }^{125}$ iodine and ${ }^{57}$ cobalt as tracers) which was purchased from Amersham International. Cholesterol was measured on the American monitor "parallel" system by an enzymatic method using cholesterol oxidase with amino antipyrine as chromogen. High density lipoprotein (HDL) cholesterol was measured by the CHOD-PAP method on a Roche "Cobas-Bio" centrifugal analyser, following precipitation of the serum with manganese/ heparin.

Smoking habit was ascertained by interviewer administered questionnaire. For the purposes of the present study subjects were classified into two categories of smoking habit; those who had never smoked and those who currently smoked or had smoked.

Dietary intake was estimated using the $7 \mathrm{~d}$ weighed inventory method. Subjects, closely supervised by fieldwork assistants, weighed (Miniscale, PC International, Little Abington, Cambridgeshire) and recorded all food and drink consumed at home; for food eaten outside the home estimated weights were used in conjunction with descriptive information. All food diaries were coded for according to McCance and Widdowson's The composition of foods ${ }^{8}$ and supplementary food tables. ${ }^{9}$ Mean daily intake in terms of $\mathbf{4 1}$ food groups was calculated using a computerised database and program.

The four dietary behaviours already described were generated using principal components analysis on the correlation matrix of the 41 food groups. ${ }^{2}$ The SPSSX statistical package (routine FACTOR) was used to identify the primary components which accounted for the main variation in dietary intake. The first four components accounted for $6 \cdot 7,6 \cdot 0,4 \cdot 7$, and $4 \cdot 1 \%$ respectively of the total variation.

For the purposes of this investigation the relationship between the dietary components and the haematological and biochemical variables was examined by calculating partial correlations using the SPSSX procedure PARTIAL CORR. The only blood measurement to exhibit a non-normal distribution was serum ferritin, which was approximately transformed before partial correlations were calculated. Partial correlations were separately computed for male and female subjects. In each case the partial correlations were controlled for age and smoking habit. These variables are known to affect nutrient intake, dietary patterns and biochemical measures. ${ }^{1} 210$

Results

Partial correlations between dietary patterns and haematological and biochemical variables are presented in tables I and II. As is clear from these tables the calculated correlation coefficients are 
Table II Partial correlation coefficients (controlled for age and smoking habit) between dietary behaviours and measurements of white blood cells, platelets, and serum lipids for men and women

\begin{tabular}{|c|c|c|c|c|}
\hline & \multicolumn{4}{|c|}{ Dietary behaviour } \\
\hline & "Traditional" & "Cosmopolitan" & "Convenience" & $\begin{array}{l}\text { "Meat and } \\
\text { two veg" }\end{array}$ \\
\hline \multicolumn{5}{|c|}{ Men } \\
\hline White cell count & 0.04 & -0.04 & -0.00 & -0.03 \\
\hline Platelets & 0.05 & -0.04 & 0.00 & 0.00 \\
\hline Cholesterol & -0.04 & -0.07 & -0.02 & -0.05 \\
\hline HDL cholesterol & -0.01 & $0 \cdot 12^{\star}$ & $0 \cdot 16 \dagger$ & $0.19 t$ \\
\hline \multicolumn{5}{|c|}{ Women } \\
\hline White cell count & $-0 \cdot 11^{\star}$ & $-0.15 t$ & -0.06 & -0.01 \\
\hline Platelets & $-0 \cdot 10^{\star}$ & $-0 \cdot 11^{\star}$ & -0.08 & -0.09 \\
\hline Cholesterol & -0.02 & $-0.11^{\star}$ & -0.03 & -0.07 \\
\hline HDL cholesterol & -0.01 & $0.21 \ddagger$ & $0.20 \ddagger$ & 0.03 \\
\hline
\end{tabular}

${ }^{\star} \mathrm{p}<0.05 ; \dagger \mathrm{p}<0.01 ; \pm \mathrm{p}<0.001$

small: however, when the sample size was taken into account a number of correlations was found to be of statistical significance. Those associations attaining statistical significance are discussed below.

In table I for men haemoglobin and serum ferritin levels showed a significant $(p<0.05$, $\mathrm{p}<0.01$, respectively) negative association with the "traditional" eating behaviour. In women transferrin saturation, a measure of iron transport supply, showed a negative association $(p<0.05)$ with the "traditional" behaviour. There were no significant correlations between the "cosmopolitan" behaviour and blood measurements of iron status in men. However, in women transferrin saturation showed a positive correlation $(p<0.05)$ with the "cosmopolitan" behaviour, while packed cell volume showed a negative association $(p<0 \cdot 05)$. The "convenience" behaviour was negatively associated $(p<0.05)$ with packed cell volume in men. In women transferrin saturation showed a positive correlation $(p<0.05)$ with the "convenience" behaviour while packed cell volume showed a negative correlation $(p<0.05)$. The "meat and two veg" behaviour was positively associated $(\mathrm{p}<0.01)$ with serum ferritin in men, while in women packed cell volume was positively $(p<0.01)$ associated with this behaviour.

Partial correlation coefficients between the dietary patterns and folate and vitamin B-12 status are also presented in table $I$. There were no significant correlations between the "traditional" dietary patterns and folate and vitamin B-12 status measurements. Similarly the "cosmopolitan" and "convenience" behaviours

Table III Ranking of major food groups within the "traditional" dietary behaviour

\begin{tabular}{ll}
\hline Foods with positive loadings & Foods with negative loadings \\
\hline (1) Bread & (1) Miscellaneous \\
(2) Spreading fats & (2) Chips \\
(3) Tea and coffee & (3) Wines, liqueurs, spirits \\
(4) Cakes and desserts & (4) Cooked meat dishes \\
(5) Potatoes & (5) Rice and pasta \\
(6) Milk & (6) Soft drinks \\
(7) Preserves & (7) Savoury pies \\
(8) Eggs & (8) Confectionery \\
(9) Bacon & \\
(10) Vegetables & \\
(11) Cheese & \\
(12) Breakfast cereals & \\
(13) Sugar & \\
(14) Canned meats & \\
(15) Meat & \\
(16) Fruit &
\end{tabular}

a Based on magnitude of factor loadings ( $>0.15)$ of various food groups derived from mean daily consumption of 592 subjects from Northern Ireland ${ }^{2}$ were not significantly associated with folate or vitamin B-12 status in men. In women, positive correlations were observed between red cells folate $(p<0.05)$, serum folate $(p<0.01)$, and serum vitamin $\mathrm{B}-12 \quad(\mathrm{p}<0.01)$ and the "cosmopolitan" behaviour. These three blood measurements also showed significant $(p<0.05$, $\mathrm{p}<0.01$, and $\mathrm{p}<0.01$ respectively) positive correlations with the "convenience" behaviour in women. In men the "meat and two veg" behaviour was positively correlated $(p<0.05)$ with serum vitamin B-12. In women there were no significant associations between the "meat and two veg" behaviour and any folate or vitamin B-12 measurement.

Table II shows the partial correlation coefficients between dietary behaviours and some biochemical indices of general health. In men white cell count and platelet levels did not show significant associations with any of the eating behaviours. In women the "traditional" behaviour was negatively associated $(p<0.05)$ with white cell count and platelets. In women the "cosmopolitan" dietary behaviour also showed negative correlations with white cell count $(p<0.01)$ and platelet levels $(p<0.05)$.

Levels of serum lipids also showed statistically significant associations with dietary behaviour. There were no significant associations with the "traditional" behaviour in either men or women. In both men and women the "cosmopolitan" behaviour was positively correlated with HDL cholesterol ( $p<0.05$ and $p<0.001$, respectively), and in women only, a negative correlation was observed for total cholesterol $(p<0.05)$. In both men and women the "convenience" diet was strongly positively correlated with levels of HDL cholesterol; the respective significance levels were $p<0.01$ and $p<0.001$. In men the "meat and two veg" behaviour was positively correlated $(\mathrm{p}<0.01)$ with HDL cholesterol, while for women, although the correlation was positive, it did not attain statistical significance.

In order that the relationships between dietary behaviour and biochemical measures may be interpreted in terms of food eaten, tables III-VI show the major foods comprising each dietary behaviour ranked by the magnitude of the factor loadings from the principal component analysis. ${ }^{2}$

\section{Discussion}

This enquiry has explored the relationship between patterns of food intake and nutritional health as defined by haematological and biochemical measures for a discrete population group in Northern Ireland. The relationship between dietary nutrient intake and blood biochemical measures within population studies are notoriously fragile ${ }^{11}$ and may be confounded by such factors as the biological variability in nutrient requirement and the known insensitivity of laboratory indicators of nutritional status. Allied to such problems, the inherent difficulties in measuring habitual food intake may limit the significance of absolute levels of nutrient intake, while the effects of nutrient interactions are impossible to quantify. In an attempt to overcome some of these problems a dietary pattern or food combination approach was adopted for this study. 
Table IV Ranking of major food groups within the "cosmopolitan" dietary behaviour
Table $V$ Ranking of major food groups within the "convenience" dietary behaviour

Table VI Ranking of major food groups within the "meat and two veg" dietary behaviour
The combination of foods that comprised the "traditional" pattern appeared to adversely affect iron status in both men and women. Although the prevalence of iron deficiency anaemia is low in Northern Ireland 12 adherents of the "traditional" behaviour tended to have low levels of haemoglobin, serum ferritin, and transferrin saturation. The latter two measurements are indicators of storage iron and iron transport supply respectively. Conversely the "meat and two veg" behaviour was positively associated with iron status, especially in the male cohort. These effects may be related to the combination of foods that comprised the behaviours.

A major component of the "meat and two veg" behaviour was the consumption of food rich in haem iron, namely meat, fish and poultry, which

\begin{tabular}{ll}
\hline Foods with positive loadings & Foods with negative loadings \\
\hline \begin{tabular}{ll} 
(1) Fruit & (1) Chips \\
(2) Vegetables & (2) Sausages \\
(3) Rice and pasta & (3) Peas and beans \\
(4) Egg and cheese dishes & (4) Savoury pies \\
(5) Cream & (5) Beer \\
(6) Cheese & (6) Bread \\
(7) Tea and coffee & (7) Potatoes \\
(8) Patés & (8) Canned meats \\
(9) Preserves & (9) Eggs \\
(10) Breakfast cereals & (11) Meat \\
(11) Wines, liqueurs, spirits & (12) Miscellaneous \\
(12) Fatty fish & (13) Spreading fats \\
(13) Cakes and desserts & (14) Bacon \\
(14) Shellfish & (15) White fish \\
(15) Cooked meat dishes & \\
(16) Milk & \\
(17) Sugar & \\
(18) Nuts & \\
(19) Yoghurt & \\
\hline a Based on magnitude of factor loadings (>0.15) of various \\
food groups derived from mean daily consumption of 592 \\
subjects from Northern Ireland
\end{tabular}
\end{tabular}

Foods with positive loadings

(1) Beer

(2) Chips

(3) Sauces

(4) Soft drinks

(5) Nuts

(6) Cheese

(7) Rice and pasta

(8) Miscellaneous

(9) Savoury pies

(10) Cooked meat dishes

(11) Peas and beans

(12) Yoghurt

(13) Potatoes

(14) Cream

(15) Milk

(16) Wines, liqueurs, spirits

(17) Egg and cheese dishes

(17) Egg and ch

(19) Sausages

20) Vegetable

(21) Shellfish

(22) Confectionery

(23) Meat

(24) Cider

25) Canned meat

a Based on magnitude of factor loadings ( $>0 \cdot 15)$ of various food groups derived from mean daily consumption of 592 subjects from Northern Ireland ${ }^{2}$

\begin{tabular}{ll}
\hline Foods with positive loadings & Foods with negative loadings \\
\hline (1) Meat & (1) Nuts \\
(2) Vegetables & (2) Cider \\
(3) Poultry & (3) Patés \\
(4) Sauces & (4) Cheese \\
(5) Potatoes & (5) Miscellaneous \\
(6) Wines, liqueurs, spirits & (6) Confectionery \\
(7) Rice and pasta & (7) Bread \\
(8) Fatty fish & (8) Yoghurt \\
(9) White fish & (9) Canned meats \\
(10) Shellfish & (10) Spreading fats \\
& (11) Tea and coffee
\end{tabular}

a Based on magnitude of factor loadings ( $>0 \cdot 15)$ of various food groups derived from mean daily consumption of 592 subjects from Northern Ireland ${ }^{2}$ are known to promote iron absorption. ${ }^{13} 14$ Although meat and bacon consumption featured within the food spectrum of the "traditional" behaviour, the effect of tannins, through the predominance of tea and coffee, would militate against optimum iron bioavailability. ${ }^{13} 14$ The respective inferiority and superiority of alcoholic beverages within the "traditional" and "meat and two veg" behaviours would support the associations with iron status, since indices of iron status are markedly affected by alcohol consumption. ${ }^{15}$

High haemoglobin and packed cell volume have also been identified ${ }^{1617}$ as risk factors for ischaemic heart disease, while low levels of these indices of iron status have been associated with increased mortality from causes other than ischaemic heart disease. ${ }^{16} 18$ It has also been shown that packed cell volume is decreased in vegetarians compared with omnivores. ${ }^{19} 20$ The positive correlation between the "meat and two veg" dietary behaviour and the negative correlation between the "cosmopolitan" and "convenience" dietary behaviour and packed cell volume are thus difficult to interpret both with regard to health status and food consumption. The relative ranking of meat within the "meat and two veg" pattern would support the observed positive association with packed cell volume in women; however meat consumption was also a feature, to a lesser extent, of the "convenience" and "cosmopolitan" patterns.

The relationship between dietary patterns and folate status was also reflected in the predominant foods of each pattern. Although folate is widely distributed in foods, it is rapidly destroyed by extensive cooking; hence raw and fresh food and vegetables are important dietary sources. The positive associations observed betweeen folate status and the "cosmopolitan" and "convenience" behaviours in women may be related to the relative hierarchy of fruit and vegetables in each behaviour. Fruit and vegetables majored within the "cosmopolitan" behaviour. Fruit was an inferior food within the "convenience" behaviour, while vegetables assumed median status. The relative proportion of vegetable consumption comprising salad vegetables as opposed to cooked green and root vegetables would be expected to influence blood folate levels. Unfortunately vegetable intake was not classified into these categories. However, given the nature of the "convenience" behaviour, a predominance of foods of low culinary complexity, ${ }^{2}$ it is likely that vegetable intake would be of the salad variety. To compound these effects the consumption of nuts, which are rich sources of folate, prevailed in both the "cosmopolitan" and "convenience" behaviours. Rodger $e t a l^{21}$ reported an association between alcohol consumption and folate status in both men and women. The effects of alcohol intake were, in part, attributed to the high folate content of beer although it was noted that women were more susceptible to the known haematological toxicity of alcohol. ${ }^{21}$ Beer consumption ranked high in the foods of the "convenience" behaviour while wines, liqueurs, and spirits were the preferred alcoholic beverages of adherents of the "cosmopolitan" behaviour. Thus it would seem that the relative ranking of the 
food aggregates of raw and fresh fruit and vegetables, nuts, and beer within eating behaviours may determine blood folate status, although the effect is not consistent between the sexes. In a similar fashion the occurrence of meat within all dietary behaviours reflected, in general, the observed positive correlations between vitamin B-12 status and dietary behaviours.

A number of epidemiological studies (see Ernst et $a l^{22}$ ) have identified white cell count as a risk marker for ischaemic heart disease. It has also been suggested that social class differences in ischaemic heart disease prevalence may be mediated through haemostatic mechanisms including white cell count levels. ${ }^{23}$ However this effect may be confounded by social class differences in smoking habits. ${ }^{23}$ In the present study both the "traditional" and "cosmopolitan" behaviours were associated with low levels of white cell count in women. These dietary effects on the white cell count were observed irrespective of smoking behaviour. There was also a marked dietary effect for serum total cholesterol, a classic risk marker for ischaemic heart disease, with women followers of the "cosmopolitan" behaviour tending to have lower levels. Although experimental studies have shown a positive relationship between intakes of saturated fatty acids and cholesterol and serum total cholesterol, within population studies of habitual $\operatorname{diet}^{24}$ generally show that these dietary factors explain only a small population of the variance in total cholesterol levels. A similar magnitude of association was found in the present study for the "cosmopolitan" behaviour in women. While it is not possible to identify specific nutrients or foods that may provoke the observed relationship, it is however clear that in women the adoption of a varied, diverse regimen in which fruit and vegetables are priority foods is associated with lower levels of a classic risk marker for ischaemic heart disease.

There were also a number of strong associations between dietary behaviour and HDL cholesterol, a subfraction of total cholesterol. The level of HDL cholesterol is known to be inversely related to ischaemic heart disease risk. ${ }^{25-27}$ Although several variables have been shown to be associated with HDL cholesterol levels it is well established that moderate alcohol consumption is an important determinant ${ }^{28-31}$ tending to raise blood concentrations.

The positive relationships observed between HDL cholesterol levels and the "cosmopolitan", "convenience", and "meat and two veg" behaviours may thus be related to the relatively high ranking of alcoholic beverages within all of these behaviours. In an epidemiological study of women in South Wales ${ }^{32}$ it was shown that both alcohol and fatty fish consumption were dietary determinants of HDL cholesterol levels. However, these variables accounted for only $3^{\circ}{ }_{0}$ of the variance in HDL cholesterol, which is a similar result to that found in the present study. The "cosmopolitan" and "meat and two veg" behaviours contained fatty fish within the food spectrum, thereby augmenting the effects of alcohol on HDL cholesterol levels.
Many previous attempts to relate dietary variables to biochemical measures have focused specifically on estimates of nutrient intake. This investigation has shown that a multivariate approach, namely principal component analysis which seeks to establish eating patterns, may be a suitable technique to circumvent the confounding effect of nutrient interactions and thus to elucidate the relations between food aggregates and nutritional and health status. Indeed, this technique has served to resolve the constellations of foods that interact to influence haematological indices of iron status as well as folate and HDL cholesterol levels.

This work was supported by the Health Promotion Research Trust. The authors wish to thank $\mathrm{Mr} \mathrm{S}$ Nesbitt and Mr D W Neill, Department of Clinical Biochemistry and $\operatorname{Dr} T$ Lappin, Department of Haematology, Royal Victoria Hospital, Belfast, for advice and help with the blood analyses.

1 Barker ME, McClean SI, McKenna PG, et al. Diet, lifestyle and health in Northern Ireland. Coleraine: University of and health in

2 Barker ME, McClean SI, Reid NG, Thompson KA. Dietary behaviours and sociocultural demographics in Northern Ireland. Br f Nutr 1990; 64: 319-29.

3 Schwerin HS, Stanton JL, Riley AM, et al. Food eating patterns and health: a re-examination of the Ten-State and HANES 1 surveys. Am $\mathcal{f}$ Clin Nutr 1981; 34: 568-80.

4 Schwerin HA, Stanton JL, Smith JL, Riley AM, Brett BE. Food, eating habits, and health: a further examination of the relationship between food eating patterns and nutritional health. Am f Clin Nutr 1988; 35: 1319-25.

5 Nicklas TA, Webber LS, Thompson B, Berenson GS. A multivariate model for assessing eating patterns and their relationship to cardiovascular risk factors: the Bogalusa Heart Study. Am f Clin Nutr 1989; 49: 1320-7.

6 Kish L. Survey sampling. New York: John Wiley, 1965: 398-401.

7 Bolton AE, Hunter WM. The labelling of proteins to high specific radioactivities by conjugation to a ${ }^{125}$ I-containing specific radioactivities by conjugation to a I-containing
acylating agent: application to the radioimmunoassay.

8 Paul AA, Southgate DAT. McCance and Widdowson's The composition of foods, 4th ed. London: HMSO, 1978.

9 Composition of foods, 4th ed. London: HMSO, 1978 . Wiles SJ, Nettleton PA, Black AE, Paul AA. The nutrient
composition of some cooked dishes eaten in Britain: a composition of some cooked dishes eaten in Britain: a 34: 189-224.

10 Strain JJ, Thompson KA, Barker ME, McKenna PG. The effect of smoking on estimates of iron status and calculated body iron stores. Tr Elem Med 1990; 7: 25-7.

11 Beaton GH. Toward harmonization of dietary, biochemical, and clinical assessments: the meanings of nutritional status and requirements. Nutr Rev 1986; 44: 349-58.

12 Strain JJ, Thompson KA, Barker ME, Carville DGM. Iron sufficiency in the population of Northern Irelandestimates from blood measurements. Br $\mathcal{f}$ Nutr 1990; 64 219-24.

13 Monsen ER. Iron nutrition and absorption: dietary factors which impact iron bioavailabilty. 7 Am Diet Assoc 1988; 88: 786-90.

14 Hallberg $\mathrm{L}$. Search for nutritional confounding factors in the relationship between iron deficiency and brain function. Am f Clin Nutr 1989; 50: 598-606.

15 Strain JJ, Thompson KA, Barker ME, McKenna PG. Alcohol consumption and measurements of iron status (abstract). Proc Nutr Soc 1989; 49: 23A

16 Campbell MJ, Elwood PC, Mackean J, Waters WE. Mortality, haemoglobin level and haematocrit in women. $\mathcal{f}$ Chron Dis 1985; 38: 881-9.

17 Knottnerus JA, Swaen GMH, Slangern JJM, Volovics A, Durinck J. Haematological parameters as risk factors for cardiac infarction, in an occupational health care setting. $f$ Clin Epidemiol 1988; 41: 67-74.

18 Elwood PC, Waters WE, Benjamin IT, Sweetman PM Mortality and anaemia in women. Lancet 1974; i: 891-4

19 Ernst E, Pietsch L, Matrai A, Eisenberg J. Blood rheology in vegetarians. Br f Nutr 1987; 56: 555-60.

20 Surapisitchat $\mathrm{T}$, Tanphaichitr V. Iron status in omnivorous and vegetarian adults. 7 Clin Biochem Nutr 1988; 4: 235-40.

21 Rodger RSC, Kletcher K, Fail BJ, Rahman H, Sviland L, Hamilton PG. Factors influencing haematological measurement in healthy adults. $f$ Chron Dis 1987; 40 : 943-7.

22 Ernst E, Hammerschmidt DE, Bagge U, Matrai A Dormandy JA. Leukocytes and the risk of ischaemic diseases. F $A M A$ 1987; 257: 2318-24.

23 Baker IA, Sweetnam PM, Yarnell JWG, Bainton D, Elwood PC. Haemostatic and other risk factors for ischaemic heor diseae and social class: evidence from the Caemic heart Speedwell Studies. Int $\mathcal{f}$ Epidemiol 1980; 17: 759-65. 
24 Berns MAM, De Vries JHM, Katan MB. Dietary and other determinants of lipoprotein levels within a population of determinants of lipoprotein levels within a population of 315 Dutch males aged 28 and 29. Eur F Clin Nutr 1990; 44

25 Gordon

Gordon T, Castelli WP, Hjortland MC, Kannel WB Dawber TR. High density lipoprotein as a protective factor against coronary heart disease. $A m f$ Med 1977; 62: 707-14.

26 Miller NE, Thelle DS, Forde OH, Mjos OD. The Tromso Heart Study, High density lipoprotein cholesterol and coronary heart disease. A prospective case-control study Lancet 1977; i: 965-8.

27 Criqui MH, Cowan LD, Tyroler HA, et al. Lipoproteins a mediators for the effects of alcohol consumption and cigarette smoking on cardiovascular mortality: results from the Lipid Research Clinic Follow-up Study. $A m$ F Epidemiol 1987; 126: 629-37.

28 Castelli WP, Gordon T, Hjortland MC, et al. Alcohol and blood lipids: the cooperative lipoprotein phenotyping study. Lancet 1985; ii: 153-5.
29 Burr ML, Fehily AM, Butland BK, Bolton CW, Eastham RD. Alcohol and high density lipoprotein cholesterol: a randomised controlled trial. Br f Nutr 1986; 56: 81-6.

30 Miller NK, Bolton CH, Hayes TM, et al. Associations of Miller NK, Bolton CH, Hayes TM, et al. Associations of alcohol consumption with plasma high density lipoprotein cholesterol and its major subfractions: the Caerphilly and Speedwell Collaborative Heart Disease Studies. $\mathcal{F}$

31 Linn S, Fulwood R, Rifkind B, et al. High density lipoprotein cholesterol levels among U.S. adults by selected demographic and socioeconomic variables. The second national health and nutrition examination survey 19761980. Am ₹ Epidemiol 1989; 129: 281-94.

32 Yarnell JWG, Milbank J, Walker CL, Fehily A, Hayes TM Determinants of high density lipoprotein and total cholesterol in women. $\mathcal{F}$ Epidemiol Community Health 1982; 36: $167-71$ 